\title{
A REFLEXIVE SPACE OF HOLOMORPHIC FUNCTIONS IN INFINITELY MANY VARIABLES
}

\author{
RAYMUNDO ALENCAR, RICHARD M. ARON AND SEÁN DINEEN
}

\begin{abstract}
We show the existence of an infinite-dimensional Banach space $E$ such that $H(E)$, the space of holomorphic functions on $E$, endowed with the $\tau_{\omega}$ topology is reflexive.
\end{abstract}

Various authors have recently discussed the problem of whether or not $\left(H(E), \tau_{\omega}\right)$ and $\left(H(E), \tau_{\delta}\right)$ are reflexive for $E$ a locally convex space. Positive results are known for nonnormed locally convex spaces (e.g. $\left(H(E), \tau_{\omega}\right)$ is reflexive when $E$ is a Fréchet nuclear DN space; (cf. 5.42 of [5])) and counterexamples have been given in the Banach space case (e.g. $\left(H\left(l^{P}\right), \tau_{\delta}\right)$ is not reflexive for any $p, 1 \leqslant p \leqslant \infty$ [7]). Necessary and sufficient conditions for the reflexivity of $\left(H(E), \tau_{\omega}\right), E$ a Banach space, are known [4] but no infinite-dimensional Banach space has been shown to satisfy these conditions.

In this paper, we remedy this situation by showing that $\left(H(U), \tau_{\omega}\right)$ is reflexive for any balanced open subset $U$ of the space $T^{\prime}$ defined by B. S. Tsirelson [8]. Indeed, it is not difficult to see that any Banach space $E$ such that $\left(H(E), \tau_{\omega}\right)$ is reflexive must have many of the properties of Tsirelson's space. Since $E^{\prime}$ is a (complemented) subspace of $\left(H(E), \tau_{\omega}\right)$, any such space $E$ would have to be reflexive. Moreover, if $l_{p} \stackrel{R}{\Leftrightarrow} E^{\prime}$ for some $p \in(1, \infty)$ then $P\left({ }^{n} E\right)$ would contain a copy of $l^{\infty}$ for $n=[q]+1$, $1 / p+1 / q=1$, via the mapping

$$
\left(a_{j}\right)_{j} \in l_{\infty} \rightarrow\left\{x \in E \rightarrow{ }^{t} R x=\left({ }^{t} R x\right)_{j} \in l_{q} \rightarrow \sum_{j=1}^{\infty} a_{j}\left({ }^{t} R x\right)_{j}^{n}\right\},
$$

where ${ }^{t} R$ is the transpose of $R$. Therefore, $E^{\prime}$ is a reflexive Banach space which does not contain any $l_{p}$. For details on the theory of holomorphic functions in infinite dimensions we refer to S. Dineen [5] and for a general survey on properties of Tsirelson's space we refer to P. G. Casazza [2].

1. $E$ will denote a Banach space over the complex numbers $\mathbf{C}$, and for any nonnegative integer $n, P\left({ }^{n} E\right)$ will be the space of continuous $n$-homogeneous polynomials on $E$ endowed with the topology of uniform convergence on the closed unit ball $B$ of $E$. For $U$ an open subset of $E, H(U)$ is the space of holomorphic

Received by the editors November 22, 1982.

1980 Mathematics Subject Classification. Primary 46G20; Secondary 32A30, 46B20.

Key words and phrases. Reflexivity, weakly sequentially continuous, Tsirelson space. 
mappings from $U$ into $\mathbf{C} . \tau_{\omega}$ is the locally convex topology on $H(U)$ generated by the seminorms ported by the compact subsets of $U$.

We shall use the following propositions.

Proposition 1 [3]. If $U$ is a balanced open subset of a Banach space with an unconditional basis, then $\left(H(U), \tau_{\omega}\right)$ is barrelled.

Proposition 2 [4]. If $U$ is a balanced open subset of a Banach space $E$ then $\left(H(U), \tau_{\omega}\right)$ is reflexive if and only if $\left(H(U), \tau_{\omega}\right)$ is barrelled and $P\left({ }^{n} E\right)$ is reflexive for each positive integer $n$.

Proposition 3 [7]. If $E$ is a Banach space such that $E^{\prime}$ has the approximation property and $n$ is a positive integer then the following are equivalent:

(1) $P\left({ }^{n} E\right)$ is reflexive,

(2) each $P \in P\left({ }^{n} E\right)$ is weakly continuous on the closed unit ball $B$ of $E$,

(3) for each $P \in P\left({ }^{n} E\right)$ there exists an $x \in B$ such that

$$
|P(x)|=\sup \{|P(y)| ; y \in B\} .
$$

Proposition 4. If $U$ is a balanced open subset of a reflexive Banach space with an unconditional basis, $E$, then $\left(H(U), \tau_{\omega}\right)$ is reflexive if and only if each continuous polynomial on $E$ is weakly sequentially continuous at the origin.

Proof. By Propositions 1, 2 and 3 the condition is necessary. We now show it is sufficient.

Let $P \in P\left({ }^{m} E\right)$ be arbitrary and suppose $\left(x_{n}\right)_{n} \subset E, x_{n} \rightarrow x$ weakly as $n \rightarrow \infty$. Let $A$ be the continuous symmetric $m$-linear form associated with $P$. Then

$$
P\left(x_{n}\right)-P(x)=P\left(x+x_{n}-x\right)-P(x)=\sum_{j=0}^{m-1}\left(\begin{array}{c}
m \\
j
\end{array}\right) A(x)^{j}\left(x_{n}-x\right)^{m-j}
$$

for all $n$.

For each $j, 0 \leqslant j \leqslant m-1$, the mapping $y \in E \rightarrow\left(\begin{array}{c}m \\ j\end{array}\right) A\left(x^{j}\right)(y)^{m-j}$ is a continuous $(m-j)$-homogeneous polynomial on $E$. Since $x_{n}-x \rightarrow 0$ weakly as $n \rightarrow \infty$, we have $\left(\begin{array}{c}m \\ j\end{array}\right) A(x)^{j}\left(x_{n}-x\right)^{m-j} \rightarrow 0$ as $n \rightarrow \infty$ for all $j, j<m$. Hence $P\left(x_{n}\right)-P(x) \rightarrow 0$ as $n \rightarrow \infty$.

Now choose $\left(y_{n}\right)_{n} \subset B$ (the closed unit ball of $E$ ) such that

$$
\left|P\left(y_{n}\right)\right| \rightarrow\|P\|=\sup _{x \in B}|P(x)| \text { as } n \rightarrow \infty .
$$

Since $E$ is reflexive, $B$ is weakly sequentially compact. Hence we can find a subsequence of $\left(y_{n}\right),\left(y_{n_{j}}\right)_{j}$, and $y \in B$ such that $y_{n_{j}} \rightarrow y$ weakly as $j \rightarrow \infty$.

Since $P$ is weakly sequentially continuous $P\left(y_{n_{j}}\right) \rightarrow P(y)$ as $j \rightarrow \infty$ and hence $|P(y)|=\lim _{j \rightarrow \infty}\left|P\left(y_{n_{j}}\right)\right|=\|P\|$. Since $P$ was an arbitrary element of $P\left({ }^{m} E\right)$, Proposition 3 implies that $P\left({ }^{m} E\right)$ is reflexive. An application of Propositions 1 and 2 now completes the proof.

2. In [8] Tsirelson described a method of constructing an interesting class of Banach spaces. We will not describe the spaces he defined but give the properties that we shall need. To conform with the notation of [ 2 and 6 ] we shall denote this space by $T^{\prime}$. 
Proposition 5 [8]. There exists a Banach sequence space (i.e. a Banach space all of whose elements are sequences of complex numbers) $T^{\prime}$ with the following properties:

(i) $T^{\prime}$ is reflexive;

(ii) the sequence $\left(u_{n}\right)_{n=1}^{\infty}, u_{n}=(0,0, \ldots, 1,0, \ldots)$ ( 1 in the nth position), is an unconditional basis for $T^{\prime}$, with unconditional basis constant 1 ;

(iii) if $N$ is any positive integer and $\left(x_{n}\right)_{n=1}^{N}$ is a sequence of vectors in $T^{\prime}$ with disjoint increasing support, $\left\|x_{n}\right\| \leqslant 1$ for all $n$, and $q^{N}\left(x_{n}\right)=0$ for all $n\left(q^{N}\right.$ is the natural projection onto the first $N$ coordinates), then $\left\|\sum_{n=1}^{N} x_{n}\right\| \leqslant 2$.

For $n$ and $m$ positive integers with $0<n<m$ we let $q_{n}=I-q^{n}$, where $I$ is the identity mapping on $T^{\prime}$ and we let $q_{n}^{m}=q^{m}-q^{n}$.

THEOREM 6. If $U$ is a balanced open subset of $T^{\prime}$ then $\left(H(U), \tau_{\omega}\right)$ is reflexive.

Proof. Suppose otherwise. Then by Proposition 4 there exist $m$ a positive integer, $P \in P\left({ }^{m} T^{\prime}\right), \delta>0$ and $\left(x_{n}\right)_{n} \subset T^{\prime}$ such that

(i) $\left\|x_{n}\right\|=1$ all $n$,

(ii) $x_{n} \rightarrow 0$ weakly as $n \rightarrow \infty$,

(iii) $\left|P\left(x_{n}\right)\right| \geqslant \delta$ for all $n$.

Choose $n_{1}$ such that $\left|P\left(q^{n_{1}} x_{1}\right)\right| \geqslant \delta / 2$. Next choose $N_{1}$ such that $\left\|q^{n_{1}} x_{j}\right\| \leqslant 1 / 2$ for all $j \geqslant N_{1}$. By induction we can choose strictly increasing sequences of positive integers $\left(n_{j}\right)_{j=1}^{\infty}$ and $\left(N_{j}\right)_{j=1}^{\infty}$ such that

$$
\left|P\left(q^{n_{j}} x_{N_{j}}\right)\right| \geqslant \delta / 2 \text { for all } j,
$$

and

$$
\left\|q^{n_{j}} x_{N_{h}}\right\| \leqslant 1 / 2^{j} \text { for all } k>j
$$

Now for any polynomial $a_{0}+a_{1} z+\cdots+a_{n} z^{n}$ of one complex variable we have $\sum_{j=0}^{n}\left|a_{j}\right|^{2} \leqslant \sup _{z \mid \leqslant 1}\left|\sum_{j=0}^{n} a_{j} z^{j}\right|^{2}$. By using this result and induction, we can find for any positive integer $l,\left(\lambda_{j}\right)_{j=2^{\prime+1}}^{2^{l+1}}$ such that

$$
\left|\lambda_{j}\right| \leqslant 1 \text { for all } j
$$

and

$$
\left|P\left(\sum_{j=2^{l}+1}^{2^{\prime+1}} \lambda_{j} q^{n_{j}}\left(x_{N_{j}}\right)\right)\right| \geqslant \frac{\delta}{2}(\sqrt{2})^{l} .
$$

On the other hand

$$
\left\|\sum_{j=2^{\prime}+1}^{2^{\prime+1}} \lambda_{j} q^{n_{j}}\left(x_{N_{j}}\right)\right\| \leqslant\left\|\sum_{j=2^{\prime}+1}^{2^{\prime+1}} \lambda_{j} q_{n_{j-1}}^{n_{j}}\left(x_{N_{j}}\right)\right\|+\left\|\sum_{j=2^{\prime}+1}^{2^{\prime+1}} \lambda_{j} q^{n_{j-1}}\left(x_{N_{j}}\right)\right\| .
$$

The sequence $\left\{\lambda_{j} q_{n_{j-1}}^{n_{j}}\left(x_{N_{j}}\right)\right\}_{j=2^{\prime}+1}^{2^{\prime+1}}$ consists of vectors with mutually disjoint support. For $2^{l}+1 \leqslant j \leqslant 2^{l+1}$ we have $n_{j-1} \geqslant j-1 \geqslant 2^{l}$ and hence

$$
q^{2^{\prime}}\left(\lambda_{j} q_{n_{j-1}}^{n_{j}}\left(x_{N_{j}}\right)\right)=0
$$


By condition (iii) of Proposition 5

$$
\left\|\sum_{j=2^{\prime}+1}^{2^{\prime+1}} \lambda_{j} q_{n_{,}-1}^{n_{1}}\left(x_{N_{j}}\right)\right\| \leqslant 2 \sup _{2^{\prime}+1 \leqslant j \leqslant 2^{l+1}}\left\|q_{n_{\prime},}^{n_{\prime}}\left(x_{N_{j}}\right)\right\| \leqslant 2 .
$$

By (2)

$$
\left\|\sum_{j=2^{\prime}+1}^{2^{\prime+1}} \lambda_{j} q^{n_{j} \cdot 1}\left(x_{N_{j}}\right)\right\| \leqslant \sum_{j=2^{\prime}+1}^{2^{\prime+1}} \frac{1}{2^{j}} \leqslant 1
$$

Hence,

$$
\left\|\sum_{j=2^{l}+1}^{2^{l+1}} \lambda_{j} q^{n_{j}}\left(x_{N_{j}}\right)\right\| \leqslant 3
$$

This implies

$$
\left|P\left(\sum_{j=2^{l}+1}^{2^{\prime+1}} \lambda_{j} q^{n j}\left(x_{N_{j}}\right)\right)\right| \leqslant\|P\| 3^{m}
$$

Hence, by (4) we have

$$
\delta(\sqrt{2})^{l} / 2 \leqslant\|P\| 3^{m}
$$

Since we can choose $l$ arbitrarily large, (6) leads to a contradiction. Hence $\left(H(U), \tau_{\omega}\right)$ is reflexive.

For more information on polynomials which are weakly sequentially continuous we refer to [1]. We note, by [1, Proposition 2.8], that every polynomial $P \in P\left({ }^{n} T^{\prime}\right)$ is a limit of finite type polynomials. Hence $P\left({ }^{n} T^{\prime}\right)$ is separable for all $n$ and $\left(H\left(T^{\prime}\right), \tau_{\omega}\right)$ is also separable. By [6], if $p=\inf \{q: T$ has lower $q$ estimates $\}$, then $T$ contains (uniformly) disjointly supported vectors which are (uniformly) equivalent to the unit vector basis of $l_{p}$. By the definition of $T$ and Theorem 3.3 of [2] we must have $p=1$. That is, $T$ has lower $p$-estimates for all $p>1$. In particular, there exists $C>0$ such that

$$
\left\|\sum_{n=1}^{\infty} \lambda_{n} t_{n}\right\| \geqslant C \cdot\left(\sum_{n=1}^{\infty}\left|\lambda_{n}\right|^{2}\right)^{1 / 2}
$$

for all $\sum_{n=1}^{\infty} \lambda_{n} t_{n} \in T$.

The mapping $\sum_{n=1}^{\infty} \lambda_{n} t_{n} \in T \rightarrow \sum_{n=1}^{\infty} \lambda_{n}^{2}$ thus defines a continuous polynomial of degree 2 on $T$ which is not weakly sequentially continuous. Hence we have the following result.

Proposition 7. The spaces $P\left({ }^{n} T\right), n \geqslant 2$, and $\left(H(T), \tau_{\omega}\right)$ are not reflexive.

Finally we remark that all our results on homogeneous polynomials on $T$ and $T^{\prime}$ can be extended by the same methods to multilinear mappings on $T$ and $T^{\prime}$.

We would like to thank P. Casazza and the referee for providing us with a direct proof and also the above short proof of Proposition 7. 


\section{BIBLIOGRAPHY}

I. R. Aron, C. Herves and M. Valdivia, Weakly continuous mappings on Banach spaces, J. Funct. Anal. 52 (1983), 189-204.

2. P. G. Casazza, Tsirelson's space, Proceedings of Research Workshop on Banach Space Theory (Bor-Luh Lin, ed.), The University of Iowa, 1981, pp. 9-22.

3. S. Dineen, Holomorphic functions on $\left(C_{0}, X_{b}\right)$ modules, Math. Ann. 196 (1972), 106-116.

4. Holomorphic functions on locally convex topological vector spaces. I, Locally convex topologies on $H(U)$, Ann. Inst. Fourier (Grenoble) 23 (1973), 19-54.

5. Complex analysis in locally convex spaces, North-Holland Math. Studies, vol. 57, North-Holland, Amsterdam, 1981.

6. J. L. Krivine, Sous-espaces de dimension finie des espaces de Banach réticulés, Ann. of Math. (2) 104 (1976), 1-29.

7. R. Ryan, Applications of topological tensor products to infinite dimensional holomorphy, Thesis, Trinity College, Dublin, 1980.

8. B. S. Tsirelson, Not every Banach space contains an imbedding of $l_{p}$ or $c_{0}$, Functional Anal. Appl. 8 (1974), 138-141.

Department of Mathematics, Universidade de SaO Paulo, CEP 05508-SaO Paulo, Brazil

Department of Mathematics, Trinity College Dublin, Dublin 2, Ireland

Department of Mathematics, University College Dublin, Belfield, Dublin 4, Ireland (Current address of Seán Dineen)

Current address (Raymundo Alencar and R. M. Aron): Department of Mathematical Sciences, Kent State University, Kent, Ohio 44242 